\title{
TOWARDS A BIO-INSPIRED MIXED-SIGNAL RETINAL PROCESSOR
}

\author{
Timothy G Constandinou ${ }^{1}$, Julius Georgiou ${ }^{1,3}$ and Chris Toumazou ${ }^{1,2}$ \\ ${ }^{1}$ EEE Dept, Imperial College of Science, Technology and Medicine, London, SW7 2BT, UK. \\ ${ }^{2}$ Toumaz Technology Limited, Culham Science Centre, Abington, Oxfordshire, OX14 3DB, UK. \\ ${ }^{3}$ Geosilicon Limited, 7 Thessalonikis Avenue, Strovolos, Nicosia 2020, Cyprus.
}

\begin{abstract}
A robust distributed architecture for real-time objectbased processing is presented for tasks such as object size, centre and count determination. This approach uses the input image to enclose a feedback loop to realize a datadriven pulsating action. Outlined is the top level design for hardware implementation in a standard CMOS technology.
\end{abstract}

\section{INTRODUCTION}

A modern advanced image processing system uses an external camera to stream the image data to the processor, executing a software algorithm. Such a modular scheme demands huge bandwidth requirements for the video transmission and therefore heavy power requirements. Several early filtering applications can benefit from combining the phototransduction and processing at the pixel level. A new breed of vision chips have recently emerged that strive to achieve precisely this. A generic reconfigurable architecture to provide such pixel-level processing is the cellular neural network processor [1]. Other systems have been inspired by the unparalleled computational efficiency of living organisms in solving complex image processing tasks. These biologicallyinspired (or retinomorphic [2]) systems have been realized to perform tasks such as image enhancement and feature extraction. Object-based processing is a fundamental task for many early vision applications. The segmentation of various objects in an image has been traditionally implemented in software using techniques such as the snake algorithm [3]. It has not been till recently, that dedicated hardware has been developed for such tasks as object-based attention selection [4] and contour length measurement [5]. This paper proposes a novel scheme [6] suitable for such object-based computation based on a distributed processing architecture. Although several of the features have been biologically inspired, the algorithm is fundamentally synthetic. By using this hybrid approach, a realistically hardware implementable system can be developed benefiting from increased computational efficiency provided by the bio-inspired analogue processing elements. The reduced power consumption enables realization of mobile diagnosis devices which would otherwise be technically unachievable.

The target application for hardware implementation is microscopic cellular population analysis as a microelectronic alternative to haemocytometry. The developed system (ORASIS) is required to provide cellular count and size information on microscopic images such as those shown in figure 1.

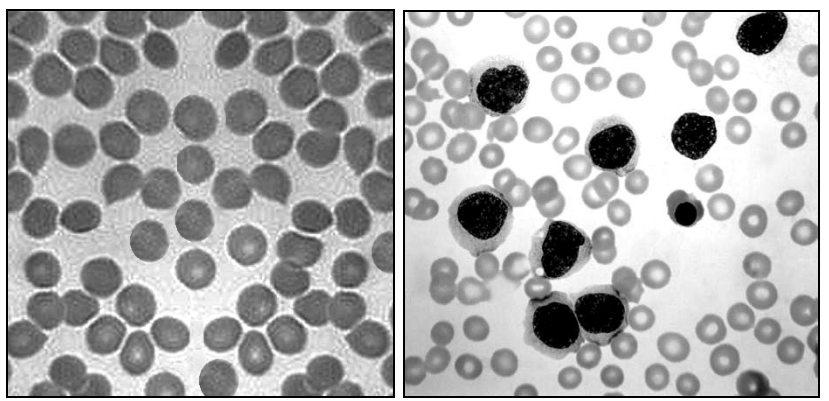

Fig 1. Sample input images of red-blood cell specimens for application in microscopic cellular population analysis

\section{ALGORITHM [6]}

A continuous-time edge-detection technique is used to form the contours and trigger the data-driven processing. On detection of an object boundary, the initial state for the signal flow is set. By propagating an inward fill, the contour can be reduced until this converges to the centre. The central point is detected by utilizing spatiotemporal integration; i.e. a summation of the cells set within the receptive field within a certain time window. On centroid detection, the object is reset and output transmitted, thus realizing an inward pulsating action. The frequency of pulsation determines the size, i.e. radius of this object. Figure 2 illustrates this interaction graphically through computer simulations.

This scheme can be applied in two different modes of operation; either as a single shot "capture and process" mode or using the above described continuous pulsating mode. The trade-off between these two modes of 
operation is accuracy (due to averaging) versus power consumption (due to increased duty cycle.)

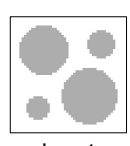

input

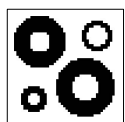

$\mathrm{t}=5$

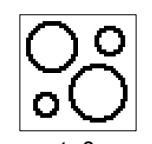

$t=0$

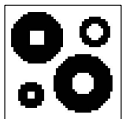

$\mathrm{t}=6$

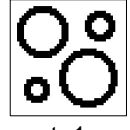

$t=1$

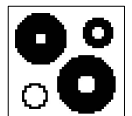

$\mathrm{t}=7$

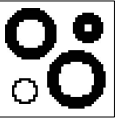

$t=2$

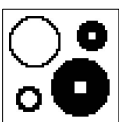

$\mathrm{t}=8$

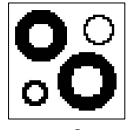

$t=3$

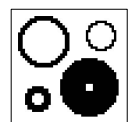

$t=9$

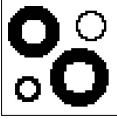

$t=4$

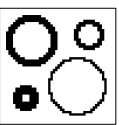

$\mathrm{t}=10$
Fig. 2 Computer simulation results of the bio-pulsating contour reduction algorithm, with snapshots taken at time intervals at the propagation delay of the processing.

\section{METHOD}

Objects are defined as regions in the image with intensity below (or above) the average level of the input image. The edges are detected by computing the difference in neighbouring cell intensities and contours are formed if a continuous edge is found; i.e. at the nodes which have two edges leading to them. The contour reduction is facilitated by setting a cells state if any of its adjacent cells have been set in addition to the object criterion being satisfied. The rate of the contour reduction is preset by introducing a delay element in the propagation cycle. The unusual feature of this method is the absence of any pre-defined synchronisation signal, for example, a clock. The only synchronisation is obtained through the data-driven object reset scheme but on a local, rather than a global basis. The reset is generated on detection of an object centre. As previously mentioned, this detection involves counting that all local pixel-cells have been set within a certain time period.

\section{BIOLOGICALLY INSPIRED APPROACH}

Many of the implemented circuits and functions have in fact been biologically inspired. As in the mammalian retina, the front-end circuitry includes continuous time logarithmic photo detection, in addition to localised smoothing (averaging) and adaptive edge detection for the signal conditioning. Furthermore, the signal propagation based on localised interaction works in a similar way to the orientation-selective V1 cells in the primary visual cortex. The centroid determination is implemented using a pseudo centre-surround receptive field technique; very similar to the functional organisation of the retina. This has been implemented using delay and propagate, integrate and fire type neuronal circuits; producing a truly spike-domain output as in the case of ON/OFF ganglion cells.

\section{HARDWARE IMPLEMENTATION}

The presented architecture is currently being realized into circuit blocks for implementation in a standard $0.18 \mu \mathrm{m}$ CMOS process provided by UMC. The circuit topology is a unique combination of both weak-inversion analogue providing micropower operation with asynchronous logic for robustness and stability.

The complete top level system architecture is shown in Figure 3. This contains an $\mathrm{X}^{*} \mathrm{Y}$ array of smart pixels; containing both the photodetecting devices and local processing circuitry. At the column and row headers are address encoders which relay the data received through a digital bus for off-chip communication. Such an encoding scheme is often referred to as address event representation (AER.) This approach is possible due to the very low output bandwidth requirement that avoids the polling of all pixels.

Containing several current-mode circuits; each pixel requires a bias current reference. A current-mode distribution scheme is adopted implementing a tree-like hierarchy. The PTAT master reference supplies the bias currents to the four corners of the array. These corner currents are then duplicated for each row and subsequently for each column, resulting in each pixel receiving an individual bias. This vastly reduces errors arising from bias current variations; a major headache when using voltage-mode current distribution. The improved current matching is due to using low-proximity current-copying circuits thus minimizing any mismatch errors; discussed in further detail in section 6 .

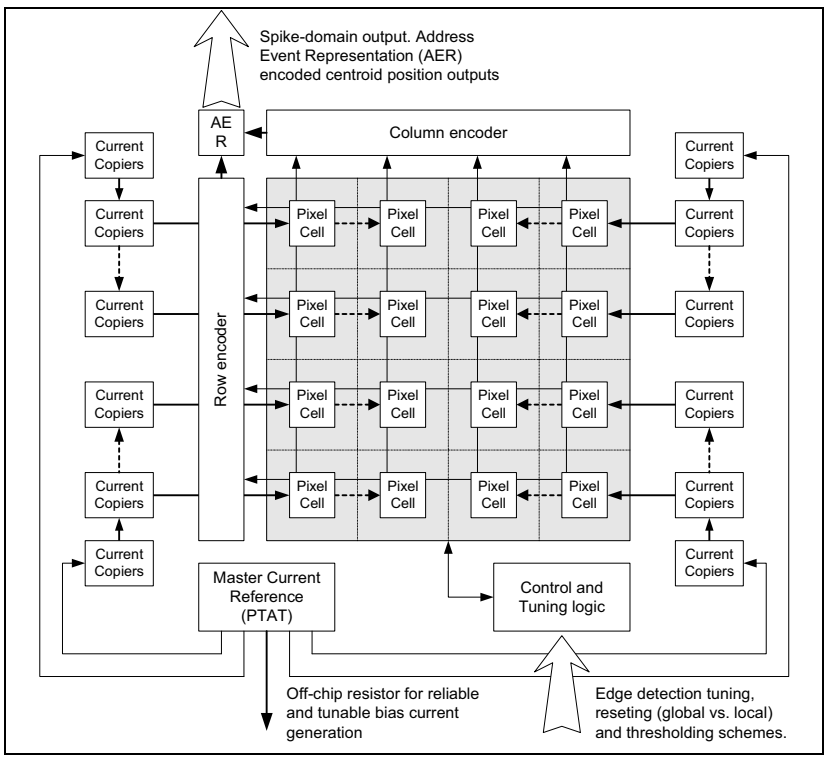

Fig. 3 Top level system architecture of an $X * Y$ array illustrating the bias distribution and output readout schemes. 
In order to facilitate the contour computation, the processing must occur at the pixel corners, as illustrated in the pixel-cell architecture shown in Figure 4.

The required functional (pixel-level) blocks; all continuous time topologies, are given below:

a. Light detection: Active photodiode (continuous time) utilizing $\mathrm{n}+$ implant $\mathrm{p}$-substrate junction diodes.

b. Edge detection: Discrete output using thresholding technique [7] utilizing differential current-mode hysteresis for computation of object contours.

c. Local averaging: Narrow-field for input image smoothing and wide-field for object detection; using current-mode circuitry for thresholding.

d. Local resetting: Dynamic switching regulated with local average current-mode thresholding [7] for object segmentation, to provide localised (object) resetting.

e. Neuromorphic logic: performing delay-and-propagate computation for signal flow and centre-surround-like computation for centroid determination.

f. Memory: Basic 1-bit memory implemented using digital (asynchronous) RS flip-flop for storage of present cellular state.

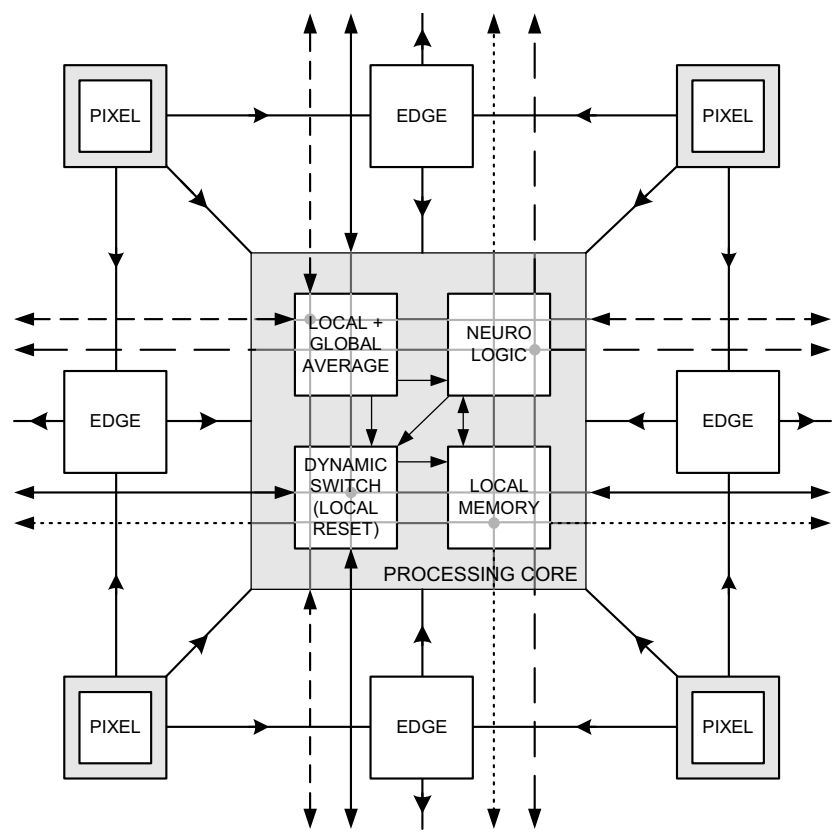

Fig. 4 Proposed cellular architecture for object-based processing illustrating organisation and connectivity of functional blocks within a quad-pixel arrangement.

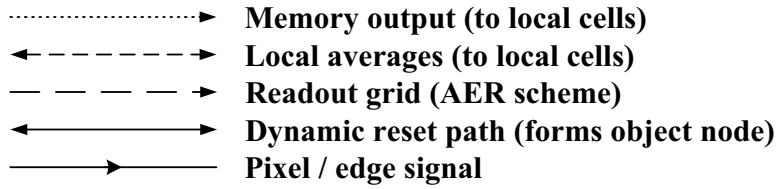

\section{DEVICE MISMATCH}

A fundamental design issue for ensuring circuits operating in weak inversion will work is device matching. The device mismatch arises from process parameter variations mainly in gate oxide thickness and doping concentrations, resulting in device threshold voltage and drain current variations. Since the $\mathrm{gm} / \mathrm{I}$ ratio is at a maximum for devices operating in weak inversion, this signifies that subthreshold circuits are those most affected by device mismatch [8].

In designing a system requiring image acquisition capabilities in standard CMOS technology, careful consideration must be taken into such sources of error. Non-uniformities in the pixel array; referred to as fixed pattern noise (FPN) are mainly due to offset and gain mismatches between the in-pixel amplifiers. This error, if uncompensated for, would normally render a processing algorithm unusable; however, the method presented has proved robust. Through computer simulations, the inherent immunity to both FPN and physical defects has been verified; discussed in section 7 . For both this reason and the high lighting conditions present in the target application, the required dynamic range is limited and therefore the FPN will not pose a serious problem.

However, mismatch errors are not limited to FPN. All circuit blocks requiring critically matched device pairs or groups are susceptible to such errors, for example differential pairs or current mirrors. Subsequently all such circuits require additional attention from schematic design through layout. Specialist simulation techniques such as Monte-Carlo analysis in additional to careful layout [9] can minimize these mismatch errors to both improve performance characteristics and production yields.

\section{SIMULATED RESULTS}

The proposed system has been simulated at all levels; from the top-level distributed algorithm, to the bottomlevel photodiode device physics. These have been facilitated using a selection of simulation tools including the Cadence Spectre Simulator and MATLAB in addition to custom developed code. For the scope of this paper, only the high-level algorithmic simulations shall be discussed.

By using the above mentioned mathematical tools, the distributed algorithm has been simulated with a wide variety of input images. Artificial fixed-pattern noise (FPN) and process defects have been introduced to demonstrate the inherent robustness and fault-tolerant properties of the contour-reduction algorithm. Through successive simulation using randomly generated noise and 
defect errors, statistical data has been compiled to illustrate a trend for the robustness and stability, given in Figure 5.
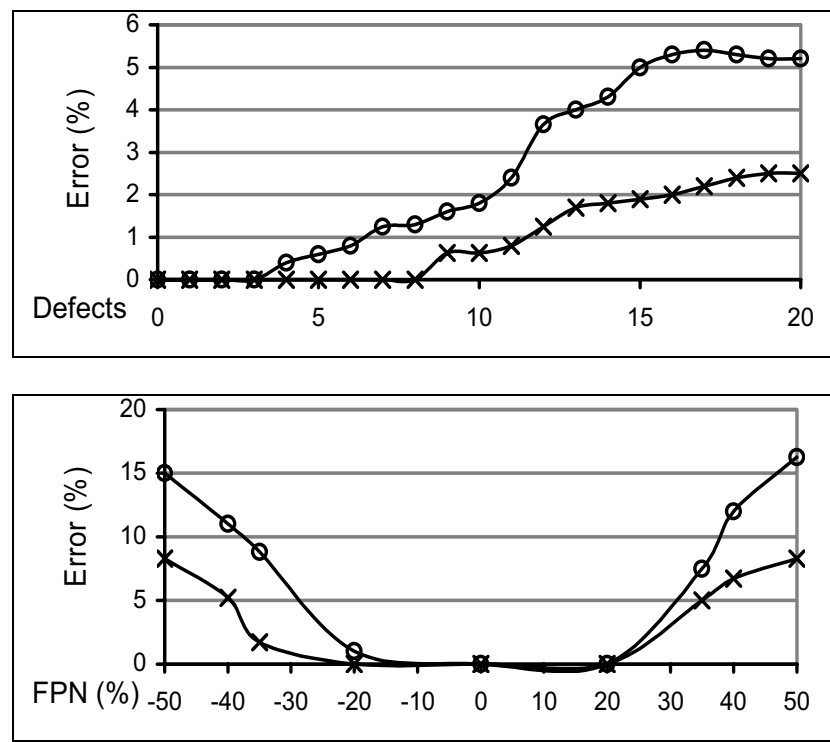

Fig. 5 Statistical data illustrating robustness to defects (top) and FPN (bottom, acquired through successive computer simulation of the bio-pulsating contour reduction algorithm.

O- Object size computation

$\longrightarrow$ Object count computation

\section{TARGET SPECIFICATIONS}

The target design specifications for the ORASIS chip are listed in table 1 .

\begin{tabular}{|c|c|c|}
\hline \multicolumn{2}{|c|}{ Technology } & UMC $0.18 \mu \mathrm{m} 1 \mathrm{P} 6 \mathrm{M}$ CMOS \\
\hline \multicolumn{2}{|c|}{ Supply voltage } & $1.8 \mathrm{~V}$ core $(3.3 \mathrm{~V} \mathrm{I} / \mathrm{O})$ \\
\hline \multicolumn{2}{|c|}{ Dynamic Range } & from $50 \mathrm{mWm}^{-2}$ to $5 \mathrm{kWm}^{-2}$ \\
\hline \multicolumn{2}{|c|}{ Responsivity } & $0.2 \mathrm{AW}^{-1} \mathrm{~m}^{-2} @ \lambda=500 \mathrm{~nm}$ \\
\hline \multicolumn{2}{|c|}{ Maximum tolerable FPN } & $+/-15 \%$ \\
\hline \multirow{7}{*}{$\frac{\Omega}{\equiv}$} & Cell Area & $90 \mu \mathrm{m} \times 90 \mu \mathrm{m}$ \\
\hline & Active fill factor & $11 \%$ \\
\hline & Pixel power & 18nW (typical) \\
\hline & Edge power & 20nW (maximum) \\
\hline & Averaging power & 95nW (typical) \\
\hline & Logic power & 5nW (maximum) \\
\hline & Total online power & $138 \mathrm{nW}$ \\
\hline \multirow{7}{*}{$\begin{array}{l}\mathscr{W} \\
\tilde{\omega} \\
\stackrel{0}{\Xi}\end{array}$} & Chip area & $25 \mathrm{~mm}^{2}$ \\
\hline & Array resolution & $40 \times 40$ cells \\
\hline & Total array power & $345 \mu \mathrm{W}$ (typical) \\
\hline & Total periphery power & $100 \mu \mathrm{W}$ (maximum) \\
\hline & Total online power & $345 \mu \mathrm{W}$ \\
\hline & Duty cycle (online) & $10 \%$ \\
\hline & Total effective power & $44.5 \mu \mathrm{W}$ \\
\hline
\end{tabular}

Table 1 Target design specifications for ORASIS cell- and system level hardware implementation

\section{CONCLUSION}

This paper outlines the top-level hardware implementation of the bio-pulsating contour reduction algorithm [6]. This is a parallel, distributed algorithm performing asynchronous object recognition breaking the bottleneck of traditional, sequential von Neuman based computational paradigm. The globally asynchronous scheme is regulated by employing data-generated local synchronisation, reducing power consumption and improving the signal-to-noise ratio. By incorporating the processing in the front end, the bandwidth requirements have been reduced by at least four orders of magnitude. Both the functionality and robustness have been verified through extensive computer simulation and by implementing an explicit architecture; the hardware realisation has been targeted for micropower operation, realising a retinal vision processor.

\section{ACKNOWLEDGEMENTS}

The authors would like to acknowledge the Basic Technology grant (UKRC GR/R87642/02) and the AMx technology grant (EPSRC GR/R96583/01,) in addition to Toumaz Technology Limited for sponsoring this research.

\section{REFERENCES}

[1] T. Roska, A. Rodriguez-Vazguez, "Towards visual microprocessors," Proc. IEEE, Vol. 90, pp. 1244-1257, 2002.

[2] K.A. Boahen, "A retinomorphic vision system," IEEE Micro, Vol. 16, pp. 30-39, 1996

[3] M. Kass, A. Witkin and D. Terzopoulos, "Snakes: active contour models," Int. J. Comput. Vision, Vol. 1, pp. 321331,1988

[4] T.G. Morris, T.K. Horiuchi and S.P. Deweerth, "Objectbased selection within an analog VLSI visual attention system," IEEE Trans. Circuits Syst. 2, Vol. 45, pp. 1564$1572,1998$.

[5] S Liu and J.G Harris, "Dynamic wires: an analog VLSI model for object processing," Int. J. Comput. Vision, Vol. 8, pp. 221-239, 1992.

[6] T.G. Constandinou, T.S. Lande, C. Toumazou, "Biopulsating architecture for object-based processing in next generation vision systems," IEE Electronics Letters, Vol. $39(16$,$) pp. 1169-1170, 2003$.

[7] T.G Constandinou, J. Georgiou and C. Toumazou, "A nanopower mixed-signal edge-detection circuit for pixellevel processing in next generation vision systems," IEE Electronics Letters, Vol. 39 (25,) pp. 1774-1775, 2003.

[8] A. Papasovic, A.G. Andreou, C.R. Westgate, "Characterisation of Subthreshold MOS Mismatch in Transistors for VLSI Systems," Analog IC's \& Signal Proc., Vol. 6, pp. 75-85, 1994.

[9] R.A. Hastings, "The Art of Analog Layout," Prentice Hall, 2000. 